\title{
Is Messier 74 a barred spiral galaxy?
}

\section{Near-infrared imaging of M 74}

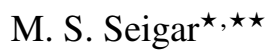 \\ Joint Astronomy Centre, 660 N. A’ohoku Place, Hilo, HI 96720, USA \\ Received 16 May 2002 / Accepted 18 July 2002

\begin{abstract}
We have obtained ground-based $I, J$ and $K$ band images of the spiral galaxy, Messier 74 (NGC 628). This galaxy has been shown to possess a circumnuclear ring of star formation from both near-infrared spectroscopy of CO absorption and sub-millimetre imaging of $\mathrm{CO}$ emission. Circumnuclear rings of star formation are believed to exist only as a result of a bar potential. In this paper we show evidence for a weak oval distortion in the centre of M 74. We use the results of Combes \& Gerin (1985) to suggest that this weak oval potential is responsible for the circumnuclear ring of star formation observed in M 74.
\end{abstract}

Key words. galaxies: individual: M74 - galaxies: fundamental parameters - galaxies: spiral - galaxies: structure

\section{Introduction}

Circumnuclear rings of star formation have been shown to occur in barred spiral galaxies since the early 1980s (e.g. Benedict 1980) and they have been studied in great detail in many galaxies since this pioneering work (e.g. Knapen 1996; Knapen et al. 1999). They are thought to be a result of a funneling of material to the central regions of the galaxies by a bar potential. Indeed, hydrodynamical simulations of galaxies have shown that gaseous material is shocked at the leading edge of a bar and diverted towards the centre of the galaxy (Roberts et al. 1979). The material can then accumulate at the inner Lindblad resonance (ILR) until it reaches a critical density at which star formation can be induced.

The spiral galaxy, Messier 74 (NGC 628), is classed as a non-barred spiral galaxy (its Hubble classification is SAc de Vaucouleurs et al. 1991). However, a circumnuclear ring of star formation does exist in the central regions of M74. This has been observed in ${ }^{12} \mathrm{CO} J=1-0$ sub-mm imaging (Wakker \& Adler 1995) and $2.3 \mu \mathrm{m} \mathrm{CO}$ absorption spectroscopy (James \& Seigar 1999). Is it therefore possible that a bar-like structure exists in the centre of M74, but is shrouded in dust? One way to answer this question is to observe M74 at near-infrared wavelengths. The first demonstration that bars are more common in the near-infrared was performed by Hackwell \& Schweizer(1983). Since then, this method has proved successful for uncovering bars in spiral galaxies in many cases (e.g. Seigar \& James 1998; Eskridge et al. 2000). In this paper we present $I, J$ and $K$ band images of M74, the longest wavelength images available, in order to uncover a bar in its centre.

\footnotetext{
* e-mail:m.seigar@jach.hawaii.edu

$\star \star$ Guest investigator of the UK Astronomy Data Centre.
}

This paper is arranged as follows: Sect. 2 describes the observations; Sect. 3 is a discussion of the results presented in this paper; in Sect. 4 we summarise our main results.

\section{Observations}

In order to uncover a bar-like structure in the centre of M 74, long wavelength images are needed. Ideally, near-infrared imaging would be the best tool for this kind of study. We have therefore retrieved archived $I, J$ and $K$ band images of M 74 .

We have made use of the Isaac Newton Group (ING) data archive to retrieve an $I$ band image of M74. The data was observed on the Jacobus Kapteyn Telescope (JKT) on 9th November 1999. Two I band images of $300 \mathrm{~s}$ each were observed. These images have been flat-fielded (using twilight sky flats) and combined to produce a single image of effective exposure time $600 \mathrm{~s}$. We also used the NASA extragalactic database (NED) to retrieve $J$ and $K$ band images of M 74. These images were observed at the Calar Alto $2.2 \mathrm{~m}$ telescope using the MAGIC NICMOS3 instrument. For each waveband, M 74 was observed for a total integration of $9 \mathrm{~min}$. These images have been flat fielded (using dome flats). The infrared images were originally presented in a paper by Möllenhoff \& Heidt (2001).

\section{Discussion}

Figure 1 shows greyscale $I, J$ and $K$ band plots of M 74 respectively. Figure 2 shows the equivalent contour plots. While it is not obvious in the greyscale plots, the $I$-band contour plot clearly shows an oval distortion in the contours at a radius of approximately 100 pixels (or 33.0 arcsec for a pixel scale 

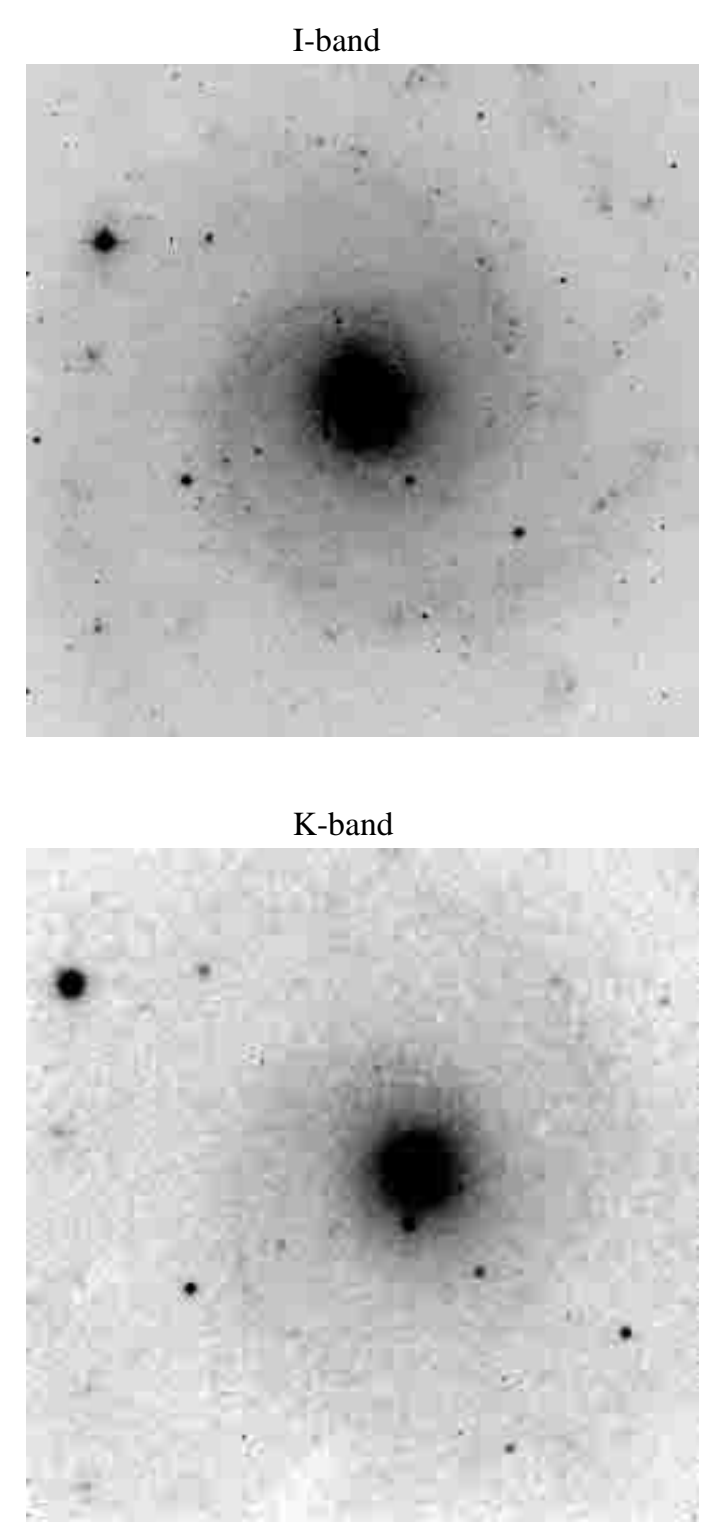

Fig. 1. $I, J$ and $K$ band greyscale plots of Messier 74 .

of $0.33 \mathrm{arcsec} / \mathrm{pixel}$ ) in the $y$-direction. The ellipticity of this contour is approximately $0.20 \pm 0.07$. The $J$-band and $K$-band images both show this elliptical contour as well, with ellipticities of $0.16 \pm 0.05$ and $0.17 \pm 0.05$ respectively. The position angle of this contour is approximately $45^{\circ}$. At a larger radius, of approximately 40-44 arcsec, the $J$ and $K$ band images also show an elliptical contour at a position angle of $-55^{\circ}$. The ellipticities of this contour are $0.28 \pm 0.05$ (in the $J$-band image) and $0.33 \pm 0.05$ (in the $K$-band image). As M 74 is a face-on galaxy, the ellipticities calculated here are real and not an inclination effect.

Figure 3 shows a plot of ellipticity of the contours shown in Fig. 2 versus minor-axis radius for both the $I$-band images (circles), the $J$-band image (triangles) and the $K$-band image (squares). Table 1 shows the same data. Both Fig. 3 and Table 1 show how the ellipticity of the $I$-band contours increases from the centre outwards and then decreases again at larger radii. The $J$ and $K$ band contours continue to increase, but their

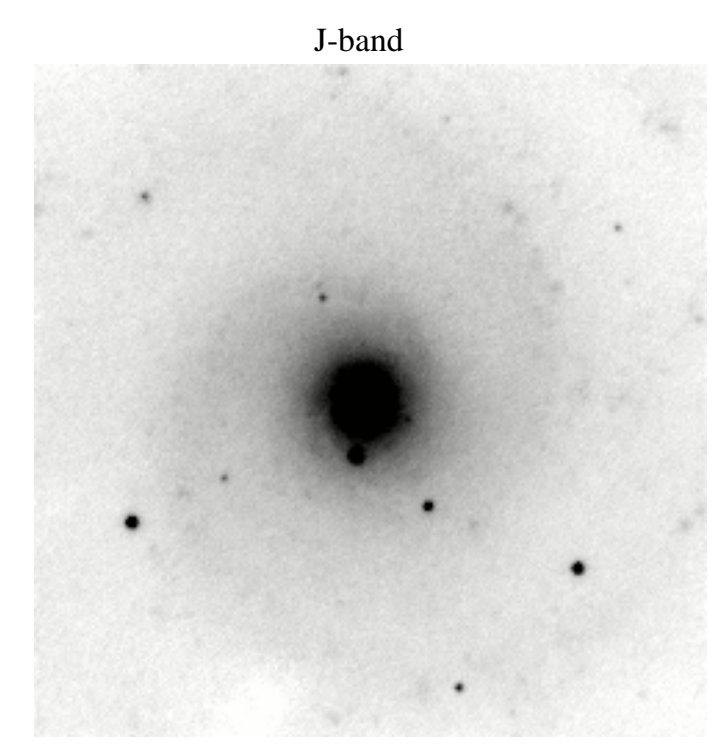

position angle changes abruptly from approximately $45^{\circ}$ to $-55^{\circ}$ between a radius of 35 and $40 \mathrm{arcsec}$. This is not seen in the $I$ band, probably due to the increase in dust extinction in this waveband. This suggests that M74 may contain a bar within a bar, i.e. a smaller scale bar which is almost perpendicular to the large scale bar. This is a common feature often seen in high resolution images of barred galaxies with circumnuclear rings of star formation (e.g. Laine et al. 2002).

What we seem to have uncovered here is an oval distortion, rather than a strong bar. Is this oval distortion strong enough to create the circumnuclear star formation ring seen in M 74?

It has been shown in simulations of barred galaxies, that the process of funnelling gas towards the central regions of galaxies by a bar, eventually leads to the self-destruction of the bar (Friedli \& Pfenniger 1991). Bar distortions are useful for driving gas towards an ILR, where the gas will then slowly accumulate. As this process continues, the gas can become gravitationally unstable and fall deeper into the potential well 

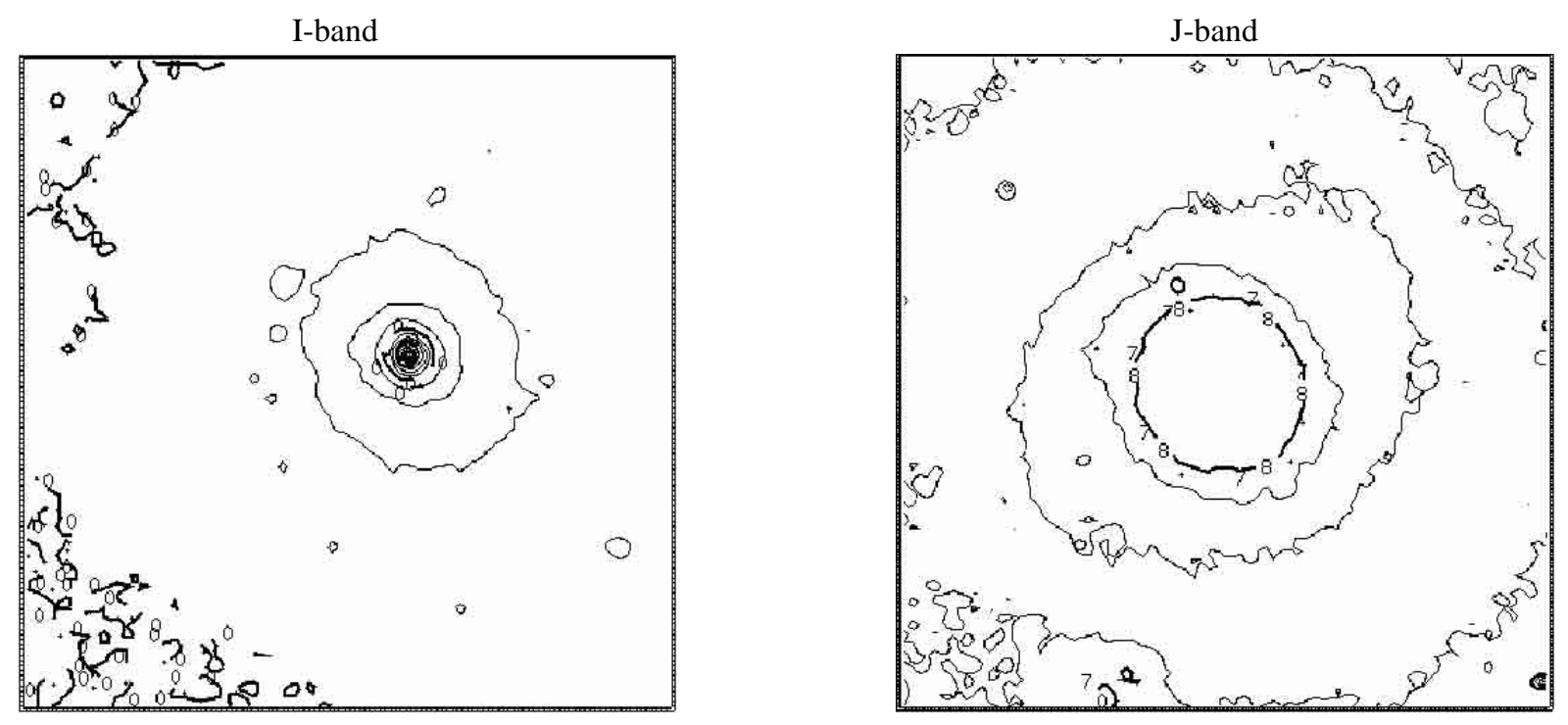

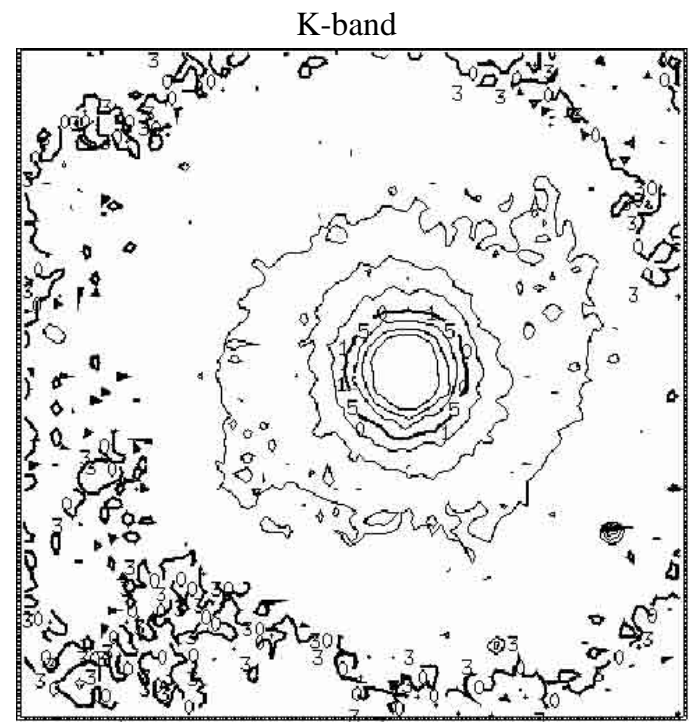

Fig. 2. $I, J$ and $K$ band contour plots of Messier 74 .

(see the double bar model in Friedli \& Martinet 1993). This accumulation of material would eventually trigger a burst of star formation, thus leading to a metal rich, red inner stellar disk. The gas could fall further inwards and create a central mass concentration. If the mass of the central accretion becomes significant an extended ILR can be formed leading to dissolution of the bar (Friedli \& Benz 1993; Friedli 1994). When the bar is dissolved signatures of its existence are often seen, e.g. boxy or peanut shaped bulges (e.g. Bureau \& Freeman 1999) or a metal rich inner stellar disk, as seen in the Sombrero galaxy (Emsellem 1995; Emsellem et al. 1996). However, these signatures are usually only observed in edge-on galaxies. It is therefore difficult to tell if the oval distortion seen in M74 is part of the progression of the dissolution of a bar.

Weliachew et al. (1988) have suggested that even a weak bar, such as the bars they infer in NGC 6946 and Maffei 2, and the bar observed in M 74 here, can affect the radial distribution of the gas. Combes \& Gerin (1985) simulated the behaviour
Table 1. Ellipticity versus radius in M 74 .

\begin{tabular}{llll}
\hline \hline $\begin{array}{l}\text { Radius } \\
(\operatorname{arcsec})\end{array}$ & $I$ band & $\begin{array}{l}\text { Ellipticity } \\
J \text { band }\end{array}$ & $K$ band \\
\hline 13.4 & - & $0.00 \pm 0.02$ & $0.00 \pm 0.02$ \\
17.2 & $0.00 \pm 0.05$ & - & - \\
23.5 & - & $0.10 \pm 0.05$ & - \\
23.8 & $0.00 \pm 0.05$ & - & - \\
28.1 & - & - & $0.17 \pm 0.05$ \\
33.0 & $0.20 \pm 0.07$ & $0.16 \pm 0.05$ & - \\
40.2 & - & - & $0.33 \pm 0.05$ \\
43.6 & - & $0.28 \pm 0.05$ & - \\
54.8 & $0.05 \pm 0.08$ & - & - \\
\hline
\end{tabular}

of an ensemble of molecular clouds in a barred galaxy, using an $N$-body barred potential and a collisional scheme for gas clouds. They used different values for the angular velocity of the bar and showed that, when this the angular velocity of the 


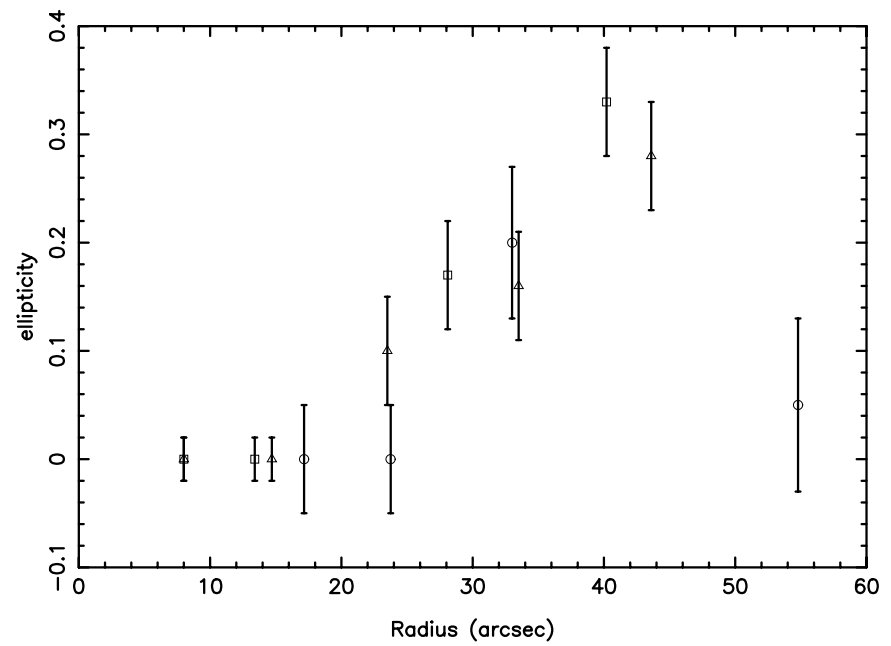

Fig. 3. Radius versus ellipticity of the isophotal contours of M 74. The circles represent the ellipticities of the isophotal contours in the the $I$ band image, triangles the $J$ band image and squares the $K$ band image.

bar is slow, spiral structure develops inside the corotation radius. Angular momentum is transferred inwards, and particles are trapped in a central orbit coincident with the ILR. Gaseous material can then accumulate at the ILR, forming a circular ring, if the bar angular velocity, $\Omega_{\mathrm{p}} \simeq(\Omega-\kappa / 2)$, where $\Omega$ is the material velocity and $\kappa$ is the epicyclic frequency. The results of the Combes \& Gerin (1985) simulations suggests that circumnuclear rings of star formation can exist, even in the presence of a weak bar potential. Thus, the oval distortion we have observed in M 74 here, may be responsible for the circumnuclear star formation activity observed by Wakker \& Adler (1995).

\section{Conclusions}

We have presented in this paper, $I, J$, and $K$ band images of the spiral galaxy, M 74. These images highlight the presence of a weak oval distortion in the central regions of the galaxy. We believe that this oval distortion is responsible for the circumnuclear ring of star formation observed in molecular $\mathrm{CO}$ emission (Wakker \& Adler 1995) and $2.3 \mu \mathrm{m} \mathrm{CO}$ absorption (James \& Seigar 1999). We have also argued that even weak oval distortions can be responsible for such circumnuclear star formation, using the simulations presented by Combes \& Gerin (1985).
Acknowledgements. This research is based on data from the ING archive. This research has made use of the NASA/IPAC Extragalactic Database (NED) which is operated by the Jet Propulsion Laboratory, California Institute of Technology, under contract with the National Aeronautics and Space Administration. The author would like to thank the anonymous referee for comments which greatly improved the content of this paper.

\section{References}

Benedict, G. F. 1980, AJ, 85, 513

Bureau, M., \& Freeman, K. C. 1999, AJ, 118, 126

Combes, F., \& Gerin, M. 1985, A\&A, 150, 327

de Vaucouleurs, G., de Vaucouleurs, A., Corwin, H. G. Jr., et al. 1991, in The Third Reference Catalog of Bright Galaxies (Univ. Texas Press, Austin)

Emsellem, E. 1995, A\&A, 303, 673

Emsellem, E., Bacon, R., Monet, G., \& Poulain, P. 1996, A\&A, 312, 777

Eskridge, P. B., et al. 2000, AJ, 119, 536

Friedli, D., \& Pfenniger, D. 1991, in Dynamics of Galaxies and Their Molecular Cloud Distributions, ed. F. Combes, \& F. Casoli (Kluwer Academic Publishers, Dordrecht), IAU Symp., 146, 362 Friedli, D., \& Benz, W. 1993, A\&A, 268, 65

Friedli, D., \& Martinet, L. 1993, A\&A, 277, 27

Friedli, D. 1994, in Mass-transfer induced activity in Galaxies, ed. I. Shlosman, 268

Hackwell, J. A., \& Schweizer, F. 1983, ApJ, 265, 643

Jaffe, W., \& van den Bosch, F. 1997, in Accretion Phenomena and Related Outflows, ed. D. T. Wickramasinghe, G. V. Bicknell, \& L. Ferrario, ASP Conf. Ser., 121, 626

James, P. A., \& Seigar, M. S. 1999, A\&A, 350, 791

Knapen, J. H. 1996, in Barred Galaxies and Circumnuclear Activity, ed. A. Sandqvist, \& P. O. Lindblad (Springer-Verlag, Berlin, Heidelberg, New York), 233

Knapen, J. H., Laine, S., \& Relaño, M. 1999, Ap\&SS, 269, 605

Laine, S., Shlosman, I., Knapen, J. H., \& Peletier, R. F. 2002, ApJ, 567, 97

Möllenhoff, C., \& Heidt, J. 2001, A\&A, 368, 16

Roberts, W. W. Jr, Huntley, J. M., \& van Albada, G. D. 1979, ApJ, 233, 67

Seigar, M. S., \& James, P. A. 1998, MNRAS, 299, 672

Wakker, P. B., \& Adler, D. S. 1995, AJ, 109, 134

Weliachew, L., Casoli, F., \& Combes, F. 1988, A\&A, 199, 29 